\title{
Aplicação de doses do herbicida glyphosate no controle da Conyza bonariensis
}

\author{
Estevan Teodoro Santana Penha* \\ Lucas Eduardo de Oliveira Aparecido** \\ Paulo Sergio de Souza*** \\ Celso Antônio Spaggiari Souza**** \\ * IFSULDEMINAS, campus Muzambinho, Discente do curso de Engenharia Agronômica. Muzambinho, Minas Gerais, Brasil. \\ estevaneafmuz@yahoo.com.br. Estrada de Muzambinho, km 35, Bairro Morro Preto, 37890-000. Muzambinho, Minas Gerais. \\ ** IFSULDEMINAS, campus Muzambinho, Discente do curso de Engenharia Agronômica. Muzambinho, Minas Gerais, Brasil. \\ lucasedap.bol@hotmail.com. (35) 91816653. Rua Virgílio Martini, 1522, Jardim Mirian, Muzambinho, Minas Gerias, cep 37890-000. \\ *** IFSULDEMIMAS, campus Muzambinho, Professor Doutor Engenheiro Agrônomo. Muzambinho, Minas Gerais, Brasil. \\ paulosergio@eafmuz.gov.br. Estrada de Muzambinho, km 35, Bairro Morro Preto, 37890-000. Muzambinho, Minas Gerais. \\ ****IFSULDEMINAS, campus Muzambinho, Professor Mestre Engenheiro Agrônomo. Muzambinho, Minas Gerais, Brasil. celso@gmail.com. \\ Estrada de Muzambinho, km 35, Bairro Morro Preto, 37890-000. Muzambinho, Minas Gerais
}

\section{Resumo}

A Conyza bonariensis é nativa da América do Sul, no Brasil tem significativa presença em campos nativos e lavouras das regiões sul, sudeste e centro-oeste. $O$ uso indiscriminado de herbicidas no manejo da lavoura provocou o surgimento de casos de resistência a eles em diversas espécies de plantas invasoras, inclusive a buva. O objetivo deste trabalho é verificar a presença de biótipos de buva resistentes ao herbicida glyphosate. O experimento foi realizado no Instituto Federal de Educação, Ciência e Tecnologia do Sul de Minas Gerais, campus Muzambinho, no setor de fruticultura. $O$ delineamento experimental foi de blocos ao acaso com quatro repetições. Os tratamentos constaram de doses crescentes de glyphosate, sendo: $0,360,720,1440,2880$ e $5760 \mathrm{~g} \mathrm{ha}^{-1}$ de ingrediente ativo. As plantas se encontravam no estádio de inflorescência. 0 experimento foi avaliado aos 21 dias após a aplicação dos tratamentos atribuindo notas com relação à toxicidade de 0 a 100\%, sendo zero a ausência de sintomas e $100 \%$ atribuído à morte da planta. Os dados percentuais foram convertidos por $\operatorname{arc} \operatorname{sen}(\sqrt{ }(\mathrm{x} / 100))$ e submetidos à análise de variância. Constatada a significância, as médias foram comparadas entre si pelo teste de Tukey ao nível de $1 \%$ de probabilidade. Os dados foram avaliados com o auxílio do programa estatístico R Development Core Team (2011). A maior dose (5760 $\mathrm{g} \mathrm{ha}^{-1}$ de ingrediente ativo) não foi capaz de promover a morte da planta (100\% de danos).

Palavras chave: Conyza spp. Resistência. Pressão de seleção. Manejo.

\section{Introdução}

A Conyza bonariensis é nativa da América do Sul, no Brasil tem significativa presença em campos nativos e lavouras das regiões Sul, Sudeste e Centro-Oeste (KISSMANN; GROTH, 1999).

A rápida dispersão da buva se deve provavelmente à grande produção de sementes. Essa espécie tem alta capacidade de formar bancos de sementes rapidamente. Cada planta produz até 150 mil sementes, que são facilmente dispersadas pelo vento (DAUER et al., 2007).

O controle da buva é realizado principalmente com a aplicação do herbicida glyphosate no manejo das lavouras, especialmente em soja e milho (VARGAS et al., 2007). O controle de plantas daninhas utilizando o método químico tornou-se o mais frequente devido à eficácia, 
facilidade de utilização e viabilidade dos custos (ABDIN, et al., 2000; JAKELAITIS et al., 2005).

No entanto, algumas plantas podem desenvolver resistência a determinado tipo de herbicida. A resistência pode ocorrer de modo natural ou pode ser induzida por engenharia genética. A resistência aos herbicidas é a capacidade de uma planta sobreviver à exposição a uma dose normalmente letal de herbicida para os indivíduos selvagens e transmitir aos seus descendentes (HEAP, 2005). A resistência é um processo natural de adaptação das plantas daninhas. Aplicações repetidas de herbicida com o mesmo espectro de ação provocam uma pressão de seleção no ambiente, fator decisivo no surgimento da resistência (CHRISTOFFOLETI, 1997; GAZZIERO; ADEGAS; VOLL, 2007).

Evidências sugerem que o surgimento de resistência a um herbicida em uma população de plantas ocorre devido à seleção de genótipos resistentes já existentes. A pressão de seleção advinda da aplicação repetida do mesmo princípio ativo estabelece condições para os genótipos resistentes se multiplicarem. O herbicida não é, portanto, o agente causador da resistência, mas sim o selecionador dos biótipos resistentes que estão em baixa quantidade no início (BETTS et al., 1992; CHRISTOFFOLETI; VICTORIA FILHO; SILVA, 1994).

Em 1980, pesquisadores japoneses foram os primeiros a relatar o caso de resistência da buva ao paraquat, também encontrado no Mississipi. No segundo caso foram encontrados biótipos resistentes à atrazina na cultura do milho em estradas e viveiros, observados em 1989 por pesquisadores belgas. Em Indiana e Ohio, foram encontrados os primeiros biótipos resistentes aos inibidores de ALS, em 1999 (LOUX et al., 2004). Mundialmente são reconhecidas 21 espécies resistentes à molécula de glyphosate, sendo que há casos de resistência da buva em 11 países (HEAP, 2005).

O herbicida glyphosate inibe a enzima 5-enolpiruvilshiquimato-3-fosfato sintase (EPSPS), responsável por converter o shiquimato-3-fosfato e o fosfoenolpiruvato em EPSP e fosfato na forma inorgânica, na rota do ácido shiquímico (GEIGER; FUCHS, 2002). A inibição desta enzima acarreta acúmulo do ácido shiquímico e redução da síntese de aminoácidos aromáticos como o triptofano, a tirosina e a fenilalanina (MOREIRA, 2008).

A resistência de biótipos de plantas daninhas a herbicidas aplicados em pós-emergência pode estar relacionada com a absorção, translocação ou exclusão diferencial dos herbicidas; com rotas de detoxificação metabólica; ou mesmo com a afinidade enzimática das moléculas. No entanto, ainda não se sabe qual é o mecanismo envolvido na resistência. Segundo Feng et al. (2004), a possível compartimentalização do produto dentro da planta e a translocação reduzida do glyphosate para os pontos de crescimento da planta e raízes são mecanismos responsáveis pela resistência. Contudo, maiores estudos devem ser realizados.

O objetivo deste trabalho é verificar a presença de biótipos de Conyza bonariensis resistentes à aplicação do herbicida glyphosate.

\section{Material e métodos}

O experimento foi realizado no período da manhã no IFSULDEMINAS (Instituto Federal de Educação, Ciência e Tecnologia do Sul de Minas), campus Muzambinho, no setor de fruticultura, em 3 de maio de 2010 , com uma temperatura de $26,8^{\circ} \mathrm{C}$. Para realização dos tratamentos foi utilizado o herbicida glyphosate em bomba costal com bico leque, com vazão de $200 \mathrm{~L} \mathrm{ha}^{-1}$ de calda. O delineamento experimental utilizado foi de blocos ao acaso com quatro repetições. Os tratamentos utilizados constaram de doses crescentes de glyphosate com $0,360,720,1440,2880$ e $5760 \mathrm{~g} \mathrm{ha}^{-1}$ de ingrediente ativo. As plantas se encontravam no estádio de inflorescência. As plantas foram avaliadas 21 dias após a aplicação dos tratamentos, atribuindo-se notas com relação à toxicidade de 0 a $100 \%$, sendo zero a 
ausência de sintomas e $100 \%$ a morte da planta. Os dados percentuais foram convertidos por $\operatorname{arc} \operatorname{sen}(\sqrt{ }(\mathrm{x} / 100))$ e submetidos à análise de variância pelo teste $\mathrm{F}$, a $0,1 \%$ de probabilidade. Nos casos em que o atributo nota foi significativo, o teste de Tukey, a $1 \%$ de probabilidade, foi utilizado para comparação das médias. Os dados foram avaliados com o auxílio do programa estatístico R Development Core Team (2011).

\section{Resultados e discussão}

Os dados analisados revelaram homogeneidade dos resíduos, aditividade do modelo e normalidade dos resíduos. $\mathrm{O}$ teste $\mathrm{F}$ (Tabela 1 ) constatou diferença entre os tratamentos empregados ao nível de 0,1\%.

Tabela 1. Análise de variância das notas atribuídas fitotoxidade do glyphosate aos biótipos de buva.

\begin{tabular}{lccccc}
\hline FV & GL & SQ & QM & F & P valor \\
\hline bloco & 3 & 0,0282 & 0,00941 & 0,3407 & 0,7962 \\
trat & 5 & 6,0539 & 1,21078 & 43,8376 & $1,995.10^{-8 * * *}$ \\
resíduo & 15 & 0,4143 & 0,4143 & & \\
\hline CV (\%) & 17,92206 & & & & \\
\hline
\end{tabular}

FV: fontes de variação; GL: graus de liberdade; SQ: soma de quadrados; QM: quadrado médio;

*** Significativo a $0,1 \%$ de probabilidade pelo teste $\mathrm{F}$.

Fonte: Elaboração própria.

Para o percentual de toxicidade avaliado, as doses de 720, 1440, 2880 e $5760 \mathrm{~g} \mathrm{ha}^{-1}$ de ingrediente ativo não obtiveram diferença mínima significativa entre si, sendo que a dose de 1440 não diferiu estatisticamente da dose de $360 \mathrm{~g} \mathrm{ha}^{-1}$ de ingrediente ativo, e todos os tratamentos diferiram da testemunha (Tabela 2).

Tabela 2. Avaliação de toxicidade, em porcentagem, provocada por doses crescentes de glyphosate aplicadas sobre um biótipo de buva (Conyza bonariensis), em condições de campo.

\begin{tabular}{cc}
\hline Tratamentos & Variável analisada \\
\hline $\begin{array}{c}\text { g ha }^{-1} \text { de ingrediente } \\
\text { ativo }\end{array}$ & Percentual de toxicidade \\
\hline 0 & $0 \mathrm{C}$ \\
360 & $30 \mathrm{~b}$ \\
720 & $90 \mathrm{a}$ \\
1440 & $72,5 \mathrm{ab}$ \\
2880 & $85 \mathrm{a}$ \\
5760 & $97,5 \mathrm{a}$ \\
\hline $\mathrm{CV}(\%)$ & 17,92 \\
\hline
\end{tabular}

* Médias seguidas de letra distintas diferem entre si pelo Teste de Tukey ao nível de 5\% de probabilidade.

Fonte: Elaboração própria.

Nenhum dos tratamentos foi capaz de promover a morte das plantas de buva, ou seja, em nenhum tratamento houve $100 \%$ de danos à planta invasora. Tal fato permite que ela ainda consiga se propagar e transmitir a sua resistência a seus descendentes. Na Figura 1 observase uma planta de buva com sintomas de intoxicação por glyphosate. Nota-se intensa brotação ao longo do caule. 
Figura 1. Planta de buva com resistência ao glyphosate.

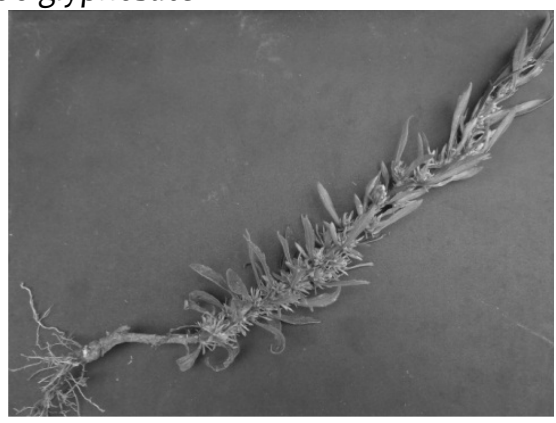

Fonte: Elaboração própria.

Vargas et al. (2006) não encontraram diferença significativa entre as doses de 2880 e 5760 $\mathrm{g}$ ha $^{-1}$ de ingrediente ativo, e obtiveram nível de toxicidade em média de $50 \%$. Estes resultados mostram que a população de plantas daninhas encontradas por esse autor se encontra sob maior pressão de seleção do que os biótipos encontrados neste trabalho.

\section{Conclusões}

A utilização do herbicida glyphosate já não é capaz de controlar a Conyza bonariensis devido à alta pressão de seleção causada pelo método hoje estabelecido de manejar a lavoura. São necessárias novas pesquisas sobre métodos de controle alternativos com outros princípios ativos e espectro de ação diferente para permitir um manejo correto que não induza à seleção de biótipos resistentes.

A maior dose de glyphosate (5760 $\mathrm{g} \mathrm{ha}^{-1}$ de ingrediente ativo) não foi capaz de controlar a buva.

\section{Application of doses of control in glyphosate herbicide horseweed}

\section{Abstract}

The Conyza bonariensis is native to South America, Brazil has a significant presence in native grasslands and crops in South, Southeast and Midwest. The indiscriminate use of herbicides in crop management gave rise to many cases of resistance to them in several weed species, including horseweed. The objective of this study is to verify the presence of horseweed biotypes resistant to glyphosate. The experiment was conducted at the Federal Institute of Education, Science and Technology South Minas Gerais, campus Muzambinho in the fruit sector. The experimental design was randomized blocks with four replications. The treatments consisted of increasing doses of glyphosate and they were $0,360,720,1440,2880$ and $5760 \mathrm{~g}$ ha-1 of active ingredient. The plants were at the stage of inflorescence. Was assessed 21 days after the treatments which scores regarding toxicity $0-100 \%$, where zero is the absence of symptoms and assigned $100 \%$ plant death. We performed data conversion percentages for arc sin $(\mathrm{V}(\mathrm{x} / 100))$ and subjected to analysis of variance, and noted the significance, the means were compared by Tukey test at $1 \%$ probability. The data were 
evaluated with the aid of the statistical program R Development Core Team (2011). The highest dose (5760 $\mathrm{g}^{-1}$ ha of active ingredient) was unable to promote death of the plant (100\% damage).

Key words: Conyza spp. Resistance. Selection pressure. Management.

\section{Referências bibliográficas}

ABDIN, O. A. et al. Cover crops and interrow tillage for weed control in short season maize (Zea mays). European Journal of Agronomy, [s.l.], v. 12, n. 2, p. 93-102, mar. 2000.

Disponível em: <http://www.sciencedirect.com/science/article/pii/s1161030199000490>. Acesso em: 28 set. 2012. DOI: http://dx.doi.org/10.1016/S1161-0301(99)00049-0

BETTS, K. J. et al. Mechanism of inheritance of diclofop resistance in Italian ryegrass (Lolium multiflorum). Weed Science, [s.l.], v.40, p.184-189, 1992.

CHRISTOFFOLETI, P. J. Resistência de plantas daninhas aos herbicidas. In: SIMPÓSIO SOBRE HERBICIDAS E PLANTAS DANINHAS, 1, Dourados - MS, 1997. Palestras... Dourados: EMBRAPA, 1997. p.75-94.

CHRISTOFFOLETI, P. J.; VICTORIA FILHO, R.; SILVA, C. B. Resistência de plantas daninhas aos herbicidas. Planta Daninha, Viçosa, v. 12, n. 1, p. 13-20, 1994.

DAUER, J. T.; MORTENSEN, D. A.; VANGESSEL, M. J. Temporal and spatial dynamics of longdistance Conyza canadensis seed dispersal. Journal of Applied Ecolology, [s.l.], v. 44, n. 1, p. 105-114, 2007.

FENG, P. C. C. et al. Investigations into glyphosate resistant horseweed (Conyza canadensis): retention, uptake, translocation and metabolism. Weed Science, [s.l.], v. 52, p. 498-505, 2004.

GAZZIERO, D. L.; ADEGAS, F. S.; VOLL, E. Indicações para o uso de glyphosate em soja transgênica. 49 ed. Londrina: Embrapa, 2007.

GEIGER, D. R; FUCHS, M. A. Inhibitors of aromatic amino acid biosynthesis (glyphosate). In: BÖGER, P.; WAKABAYASHI, K.; HIRAI, K. Herbicide classes in development. Berlin: Springer-Verlag, 2002. p. 59-85.

JAKELAITIS, A.; et al. Efeitos de herbicidas no consórcio de milho com Brachiaria brizantha. Planta Daninha, Viçosa, v.23, n. 1, p.69-78, jan./mar. 2005. Disponível em: <http://www.scielo.br/scielo.php?script=sci_arttext\&pid=S0100-83582005000100009>. Acesso em: 28 set. 2012. DOI: http://dx.doi.org/10.1590/S0100-83582005000100009

KISSMANN, K. G.; GROTH, D. Plantas infestantes e nocivas. 2 ed. São Bernardo do Campo: Basf, 1999. p. 278-284.

LOUX, M. et al. Biology and management of horseweed. 2004. Disponível em: <http://www.ipm.uiuc.edu/pubs/horseweed.pdf>. Acesso em: 26 jul. 2012. 
MOREIRA, M. S. Detecção, crescimento e manejo químico alternativo de biótipos das espécies de Buva Conyza canadensis e $C$. bonariensis resistentes ao herbicida glyphosate. 2008. 73 p. Dissertação (Mestrado). Universidade de São Paulo (Escola Superior de Agricultura "Luiz de Queiroz"), Piracicaba.

R DEVELOPMENT CORE TEAM. R Development Core Team. R: a language and environment for statistical computing. Vienna: R Foundation for Statiscal Computing, 2011. Disponível em: <http://www.R-project.org/>. Acesso em: 28 set. 2012.

VARGAS, L. et al. Resistência de Conyza bonariensis ao herbicida glyphosate. In: CONGRESSO BRASILEIRO DE CIÊNCIA DAS PLANTAS DANINHAS, 25, 2006, Brasília. Resumos... Londrina: Sociedade Brasileira da Ciência das Plantas Daninhas, 2006. p. 540.

VARGAS, L. et al. Buva (Conyza bonariensis) resistente ao glyphosate na região Sul do Brasil. Planta Daninha, Viçosa, v. 25, n. 3, p. 573-578, jul./set. 2007. Disponível em:

<http://www.scielo.br/scielo.php?pid=s0100-83582007000300017\&script=sci_arttext>. Acesso em: 28 set. 2012. DOI: http://dx.doi.org/10.1590/S0100-83582007000300017

HEAP, I. Criteria for confirmation of herbicide-resistant weeds: with specific emphasis on confirming low. [s.l.]: Weed Science, 2005. Disponível em:

$<\mathrm{http}$ ///www.weedscience.org/resgroups/Detect\%20Resistance.pdf>. Acesso em: 28 set. 2012 .

Histórico editorial

Recebido: 08/08/2012

Avaliação e copidesque: 20/08/2012 a 08/10/2012

Publicação aprovada: 30/10/2012 